\title{
Attributional style and depression
}

\author{
Harriet A. Ball, Peter McGuffin and Anne E. Farmer
}

\section{Background}

Few studies have examined whether attributional style (an individual's explanation of why events happen) is a genetically influenced vulnerability factor for depression.

\author{
Aims \\ To investigate whether attributional style is an enduring \\ vulnerability trait for recurrent depression.
}

\section{Method}

As part of the Cardiff Depression Study, we interviewed 108 people with depression and their siblings, and a control group of 105 healthy individuals and their siblings, using the Schedules for Clinical Assessment in Neuropsychiatry and the Life Events and Difficulties Schedule. Participants also completed the Attributional Style Questionnaire.

\section{Results}

Regression analyses showed that attributional style results from mood state and is not a familial risk factor for depression. However, the tendency to internalise negative events was related to having had a prior episode of depression, suggesting a 'scarring' effect. Also, non-severe events were associated with one subset of optimistic attributions.

\section{Conclusions}

Attributional style mainly measures current mood and does not reflect a familial risk factor for depression.

\section{Declaration of interest}

None. Funding from the Wellcome Trust.
In psychological research, cognitive styles have been investigated as vulnerability factors for depression onset, ${ }^{1}$ especially when attributions are in response to negative life events. ${ }^{2}$ (Abramson et al, 1989). For example, people who regard the causes of negative events as global, stable and internal (due to the self) are more likely to develop future depression. ${ }^{3,4}$ One recent study has shown genetic influences on attributional style in a population sample of adolescent twins. ${ }^{5}$ Consequently, attributional style might partly mediate genetic influences on depression. This study examines the relationship between attributional style and depression using a sib-pair design in an adult clinical sample. ${ }^{6}$ Our hypothesis was that attributional style is a familial and trait-like measure related to the genetic risk of developing depression, and is not merely associated with mood state.

\section{Method}

The UK Cardiff Depression Study has been described in detail elsewhere, ${ }^{6}$ but in brief the sample selection and interviews were as follows.

\section{Proband and sibling recruitment}

We recruited 108 people with depression following regular reviews of admissions to psychiatric clinics in Gwent and South Glamorgan and of general practitioner lists in Cardiff, Wales. Each of these participants had to have a sibling who agreed to be studied. The probands were 18-65 years old and met ICD-10 criteria for major depression, ${ }^{7}$ and were excluded if they had lifetime-ever psychotic or bipolar symptoms, or did not have a sibling willing to be studied. A control group of 105 age- and gender-matched participants was recruited from dental and orthopaedic out-patient clinics and from employees of the University Hospital of Wales National Health Service Trust (Cardiff); individuals were included if they had no current or past history of depression, and had a sibling willing to be studied. The sibling nearest in age was recruited where possible for both groups, otherwise the sibling next nearest in age was recruited. Most interviews were conducted face-to-face, but for $18 \%$ of depression group siblings and 33\% of control group siblings telephone interviews were undertaken.

\section{Interviews and questionnaires}

All participants were interviewed using the Schedules for the Clinical Assessment of Neuropsychiatry (SCAN) and the Life Events and Difficulties Schedule (LEDS). ${ }^{8,9}$ The LEDS method was used to examine life events occurring over a 12-month period prior to interview, except for participants who were currently depressed, for whom life events were assessed from 12 months prior to the onset of the current episode. An expert panel contextually rated each participant's events and difficulties. This method had good reliability. ${ }^{10}$ All participants also completed a number of self-report measures, including the Beck Depression Inventory (BDI), ${ }^{11}$ which measures depressive symptoms over the past week with good internal consistency, ${ }^{12}$ and the Attributional Style Questionnaire (ASQ).$^{13}$ The ASQ requires participants to imagine six positive and six negative hypothetical events happening to themselves. They must then nominate the major cause of the event and rate the cause along three attributional dimensions: internality ('Int'; due to something about themselves, rather than something about other people or circumstances); stability ('Stab'; due to stable rather than transient factors); and globality ('Glob'; causes that are always present rather than causes that occur once or infrequently). This gives three sub-scales each for positive (Pos) and negative (Neg) events (Int-Pos, Int-Neg, Stab-Pos, Stab-Neg, Glob-Pos and Glob-Neg). Composites for the positive (ASQ-Pos) and negative (ASQ-Neg) scales are created by summing the three sub-scales for the relevant type of event. These scales and composites have high cross-situational consistency and test-retest reliability. ${ }^{14}$

Information from the SCAN interview was entered into the CATEGO 5 scoring system to obtain ICD-10 diagnoses. ${ }^{7}$ All participants in the depressed proband group had depression of at least moderate severity according to ICD-10 criteria, indicating that their condition had reached 'caseness.'

\section{Statistical method}

Stata Statistical Software, version 9.1 for Windows, was used to create a database and perform statistical analyses. Analyses were corrected for non-independence across siblings where appropriate. 


\section{Results}

\section{Demographic details and attributional style}

The demographic details of the participating groups have been examined elsewhere. ${ }^{6}$ Briefly, there was no significant age or gender difference between the groups: depression proband group, 35\% men, mean age 39.8 years (s.e.m.=1.0); depression siblings group, $31 \%$ men, mean age 38.6 years (s.e.m.=1.0); control proband group, $26 \%$ men, mean age 36.2 years (s.e.m.=1.2); control siblings group, $40 \%$ men, mean age 39.1 years $($ s.e.m. $=1.2)$. There was no significant group difference in marital status; however, $82 \%$ of the control proband group were currently employed, compared with $52 \%$ of the depression proband group, because an excess of the latter group were registered disabled owing to their illness.

The depression proband group included 37 people who were experiencing their first depressive episode, and 71 who had also been depressed previously. Among the depression siblings group, 19 had been depressed in the past and 9 were currently depressed. Among the control group siblings, 7 had been depressed in the past but none was depressed at the time of the study.

Attributional style was not associated with age or gender, except for small influences of age on the Int-Neg sub-scale (Pearson's $r=0.10, P=0.05$ ) and of gender on the Stab-Pos subscale $(t=2.06$, d.f. $=369, P=0.04$; male mean score 29.6 , female mean score 28.4).

\section{Attributional style and current mood}

All of the ASQ scales were significantly correlated with current depression symptoms as measured by the BDI: negative scales $r=0.19$ to 0.38 , positive scales $r=-0.31$ to -0.15 ; all $P<0.01$. Four of the correlations remained significant even when examined in participants with no history of depression (Int-Pos $r=-0.16$, $P=0.02$; Glob-Neg $r=0.15, \quad P=0.02$; Stab-Neg $r=0.16, \quad P=0.01$; Stab-Pos $r=-0.11, P=0.05)$.

The mean ASQ scale scores and standard deviations for the four study groups are shown in Table 1. There was a significant overall effect of group on attributional style for all of the subscales and composites except for the Glob-Pos scale $(F=1.98$, d.f. $=3,205, P=0.12)$. When $t$-tests were undertaken, scores for the depression proband group were significantly different from those of the control probands (scores ranged from 2.32 to 6.41 , d.f. $=180$, all $P<0.01$ ). However, there was no significant difference between the two sibling groups (omitting participants who had ever been depressed, in order to detect effects of underlying familial tendencies on later depression, not associations that may be outcomes of depression): $t$ ranged from -0.43 to 1.80 , d.f. $=155-157$, all $P>0.05$. This suggests a lack of strong familial influence on attributional style.

\section{Attributional style and past depression}

In order to eliminate the effect of present mood on attributional style, we examined ASQ scores in 281 participants who had never had depression, and 26 participants who had had depression in the past but were currently mentally healthy. None of the mean scores differed between these groups.

We used the depression proband group to compare ASQ scale mean scores in participants experiencing their first episode of depression $(n=34)$ with those experiencing a recurrent episode $(n=71)$. We found no difference in the mean scores except for the Int-Neg sub-scale, for which the mean score of those with recurrent depression was 29.62 and the mean score of those for whom it was a first episode was $26.44(t=-2.12$, d.f. $=76$, $P=0.04)$. This suggests that people with recurrent depression attribute negative events to internal causes more than people experiencing their first episode of depression.

\section{Attributional style and life events}

There were weak correlations between some of the attributional style scales and life events, both for events rated as 'severe and threatening' (LEDS score 1 or 2): ASQ-Pos $r=-0.11, P=0.05$; Int-Pos $r=-0.15, P=0.01$; Glob-Neg $r=0.12, P=0.02$; Stab-Pos $r=-0.14, P=0.01$; and for events rated as 'non-severe' (LEDS score 3 or 4): ASQ-Pos $r=0.14, P<0.01$; Glob-Pos $r=0.17, P<0.01$.

\section{Familiality}

We tested for positive correlations across siblings, which would suggest genetic or shared family environmental influences on attributional style. Only Stab-Pos had a significant positive correlation ( $r=0.15$, one-tailed $P=0.027$ ). Significant findings for the other attributional style scales were absent despite the study having $80 \%$ power to detect moderate correlations $(r \sim 0.2, \alpha=0.05$, one-tailed test).

Table 1 Attributional style mean scores according to group, and one-way analysis of variance for group differences

\begin{tabular}{|c|c|c|c|c|c|c|c|}
\hline & \multicolumn{4}{|c|}{ ASQ score, mean (s.d.) } & & & \\
\hline & \multicolumn{2}{|c|}{ Depression group } & \multicolumn{2}{|c|}{ Control group } & \multicolumn{3}{|c|}{ One-way ANOVA, d.f. $=3$} \\
\hline & Probands & Siblings & Probands & Siblings & $F$ & d.f. (model, error) & $P$ \\
\hline ASQ-Neg ${ }^{a}$ & $81.4(15.2)$ & 72.5 (15.2) & $68.0(13.3)$ & $70.3(13.7)$ & 13.96 & 3,205 & $<0.01$ \\
\hline ASQ-Pos ${ }^{b}$ & 78.5 (19.8) & $84.2(14.3)$ & $85.9(13.8)$ & $85.2(17.5)$ & 2.76 & 3,205 & 0.04 \\
\hline \multicolumn{8}{|l|}{ Sub-scales ${ }^{c}$} \\
\hline Int-Neg & $28.5(6.4)$ & $26.6(6.7)$ & $24.4(5.6)$ & $26.0(5.9)$ & 7.45 & 3,205 & $<0.01$ \\
\hline Int-Pos & $25.8(7.0)$ & $29.1(5.6)$ & $28.9(6.0)$ & 30.1 (5.2) & 7.03 & 3,205 & $<0.01$ \\
\hline Glob-Neg & $25.7(7.5)$ & $20.8(7.3)$ & $19.1(6.4)$ & $19.6(6.7)$ & 15.17 & 3,205 & $<0.01$ \\
\hline Glob-Pos & $25.4(7.3)$ & $26.6(7.3)$ & $27.8(6.7)$ & $27.3(7.0)$ & 1.98 & 3,205 & 0.12 \\
\hline Stab-Neg & $27.8(5.4)$ & $25.4(5.7)$ & $23.9(5.3)$ & $24.3(5.3)$ & 9.38 & 3,205 & $<0.01$ \\
\hline Stab-Pos & $27.0(6.0)$ & $28.6(5.2)$ & $29.4(5.2)$ & $29.9(5.2)$ & 4.17 & 3,205 & $<0.01$ \\
\hline \multicolumn{8}{|c|}{$\begin{array}{l}\text { ANOVA, analysis of variance; ASQ, Attributional Style Questionnaire; Glob, global; Int, internal; Neg, negative; Pos, positive, Stab, stability. } \\
\text { a. Composite of Int-Neg, Glob-Neg and Stab-Neg sub-scales. } \\
\text { b. Composite of Int-Pos, Glob-POs and Stab-Pos sub-scales. } \\
\text { c. Int-Nes, sub-scale of internal attributions towards negative events; Int-Pos, sub-scale of internal attributions towards positive events; Glob-Neg, sub-scale of global attributions } \\
\text { towards negative events; Glob-Pos, sub-scale of global attributions towards positive events; Stab-Neg, sub-scale of stable attributions towards negative events; Stab-Pos, sub-scale } \\
\text { of stable attributions towards positive events. }\end{array}$} \\
\hline
\end{tabular}




\section{Multiple regression analysis}

Regression models were applied in order to assess the main predictors of ASQ scales: ASQ-Neg, ASQ-Pos, Int-Neg, Int-Pos, Glob-Neg, Glob-Pos, Stab-Neg and Stab-Pos were used as dependent variables in eight separate regression models. In total 143 of the participants had been treated for depression, including 117 who were currently depressed and 26 who were currently well but had had depression in the past. These categories were used to create two dummy variables. In addition, two further dummy variables were used: whether the participant was a proband or a sibling, and whether the participant was from a depressed sibling pair or a control sibling pair. These four dummy variables, along with BDI score (as a measure of current depression), gender, age, number of severe and threatening life events in 1 year (LEDS rated 1 or 2) and number of non-severe life events in 1 year (LEDS rated 3 or 4 ), were entered into the analyses as independent variables. All independent variables were included in backwards method multiple regression analyses with the significance level of $P$ to remove set at $\geqslant 0.05$. The results are shown in Table 2 .

The major significant predictor of attributional style subscales and composite scores was self-reported current mood (BDI). It remained in the final step of the backwards regression in all models except Int-Neg and ASQ-Pos. In Int-Neg and ASQ-Pos the presence of the 'currently depressed' and the absence of the BDI variables suggest that objectively defined depression (from the SCAN interview) is a better predictor of attributional style than is self-reported current mood (from the BDI). For the other scales, BDI is a better predictor than diagnosed depression.

There was an association between number of less severe life events and the composite ASQ-Pos scale $(\beta=0.14, P=0.005)$, independent of depression status. This effect came mainly from the Glob-Pos sub-scale, which had a $\beta$ of $0.17(P=0.001)$ for less severe life events. Participants who had more non-severe life events were more likely to rate positive events as due to global factors. Severe life events did not remain as significant independent predictors of any of the attributional style scales.

Gender predicted the ASQ-Neg and ASQ-Pos composites, and the Stab-Neg sub-scale. Women rated both negative and positive events as less likely to be due to internal, stable or global causes.

The proband/sibling variable remained in the model for IntNeg $(\beta=0.14, P=0.015)$, when the only other remaining predictor was current depression. This indicates that the control group probands were slightly less pessimistic than the rest of the currently mentally healthy participants. This is probably a reflection of the control proband group being 'extra healthy' owing to the process of selecting these participants for mental health.

\section{Discussion}

Past work in psychology has suggested that cognitive attributions are vulnerability factors that alter the risk of developing depression. Contrary to this hypothesis, our study suggests that attributional style is mainly an outcome of current mood, rather than a habitual (trait-like) mode of thinking that confers susceptibility to clinical depression. This was shown by the stronger relationship of attributional style with current mood (as measured by the Beck Depression Inventory) than with either a current clinical diagnosis of depression or a past history of depression (which are indices of 'trait' depression). Vulnerability factors are generally assumed to be trait-like, but here attributional style has been shown to be considerably state-like. This implies that previous studies that have examined the relationship between earlier attributions and later depressive episodes might have overlooked confounding by mood at the earlier time point, even if the participants were not yet clinically affected. However, one component of attributional style, that of attributing negative events to personal (internal) characteristics (Int-Neg), was associated with a diagnosis of depression rather than just with current mood, and this was especially so among currently depressed participants who had had previous episodes of depressive disorder. Thus, Int-Neg can be conceptualised as a 'scar' effect characterising those with recurrent disorder. In contrast, current mood (measured by BDI) was a better predictor of attributional style in all the other five sub-scales. This suggests that overall, attributional style is a reflection of current mood rather than a prior cause of depression.

Certain facets of personality are strongly associated with major depression, ${ }^{15}$ and one explanation is that personality is a vulnerability factor for depression. Attributional style is closely linked to cognitive aspects of personality, and in a previous study of this sample we found that neuroticism and extroversion were also strongly related to current mood states. ${ }^{6}$ Other studies have shown that much of the association between neuroticism and depression is due to aetiological risk factors that influence both characteristics, more than the effects of phenotypic causation (i.e. earlier neuroticism directly causing later depression). ${ }^{16}$ Similarly, attributional style may be associated with depression due to factors in the environment that predispose to both negative attributions and

Table 2 Multiple regression of attribution style scales, showing only the independent variables remaining at the final step of backwards regression $(P<0.05)$

\begin{tabular}{|c|c|c|c|c|c|}
\hline \multirow[b]{2}{*}{ Dependent variable } & \multicolumn{5}{|c|}{ Beta weights (probability) of independent variables } \\
\hline & Current depression & BDI & Gender & PROBSIB & Non-severe life events ${ }^{a}$ \\
\hline ASQ-Neg ${ }^{\mathrm{b}}$ & & $0.36(P<0.001)$ & $-0.13(P=0.010)$ & & \\
\hline ASQ-POS ${ }^{c}$ & $-0.18(P=0.001)$ & & $-0.10(P=0.036)$ & & $0.14(P=0.005)$ \\
\hline \multicolumn{6}{|l|}{ Sub-scales $^{d}$} \\
\hline Int-Neg & \multirow[t]{2}{*}{$0.29(P<0.001)$} & \multirow{2}{*}{$-0.31(P<0.001)$} & \multirow{6}{*}{$-0.15(P=0.005)$} & \multirow[t]{6}{*}{$0.14(P=0.015)$} & \multirow{6}{*}{$0.17(P=0.001)$} \\
\hline Int-Pos & & & & & \\
\hline Glob-Neg & $0.16(P=0.040)$ & $0.26(P<0.001)$ & & & \\
\hline Glob-Pos & & $-0.14(P=0.004)$ & & & \\
\hline Stab-Neg & & $0.33(P<0.001)$ & & & \\
\hline Stab-Pos & & $-0.22(P<0.001)$ & & & \\
\hline $\begin{array}{l}\text { ASQ, Attributional Style } \\
\text { a. Rated } 3 \text { or } 4 \text { on the Lif } \\
\text { b. Composite of Int-Neg, } \\
\text { c. Composite of Int-Pos, } \\
\text { d. Int-Neg, sub-scale of } \\
\text { towards negative events; } \\
\text { of stable attributions tow }\end{array}$ & $\begin{array}{l}\text { Beck Depression Inventory } \\
\text { ulties Schedule. } \\
\text { b-Neg sub-scales. } \\
\text {-Pos sub-scales. } \\
\text { towards negative events; } \\
\text { ale of global attributions to } \\
\text { ts. }\end{array}$ & $\begin{array}{l}\text { Glob, global; Int, interna } \\
\text { it-Pos, sub-scale of inte } \\
\text { ards positive events; Stc }\end{array}$ & $\begin{array}{l}\text { eg, negative; Pos, posi } \\
\text { attributions towards p } \\
\text { veg, sub-scale of stable }\end{array}$ & $\begin{array}{l}\text { ROBSIB, 'proband } \\
\text { e events; Glob-Ne } \\
\text { butions towards n }\end{array}$ & $\begin{array}{l}\text { bling' status; Stab, stability. } \\
\text {-scale of global attributions } \\
\text { e events; Stab-Pos, sub-scale }\end{array}$ \\
\hline
\end{tabular}


diagnosed depression, but the attributions themselves may not have a direct role in the development of depression.

\section{Aetiological influences on attributional style}

Severe life events did not appear to influence attributional style, once current mood and diagnosed depression were taken into account. Independent of current mood and depression status, one sub-scale of attributional style (Glob-Pos) was related to the experience of non-severe life events, in such a way that experiencing minor difficulties makes one less likely to have a pessimistic attributional style. This may reflect a causal association in which minor life events are protective against pessimistic attributions. We found similar 'protective' effects of non-severe life events in this sample when examining other potential vulnerability factors for depression, including the experience of severe and threatening life events and sensation-seeking. ${ }^{17,18}$

We found support for only one out of the six attributional style sub-scales being a potential mediator of the genetic factors in depression: only Stab-Pos had a small correlation across siblings. This correlation of 0.15 indicates at most a heritability $\left(h^{2}\right)$ of $30 \%$, calculated as $r_{\mathrm{sib}}=1 / 2 h^{2}$ (if we assume negligible influence of shared family environment). The other scales did not correlate significantly across siblings. This indicates strong influences from non-shared environmental factors (those not shared across siblings). Life events were not a predictor of attributional style independent of depression status, but life events could still account for some of the non-shared environmental influence on attributions if mediated by onset of a depressive episode.

The lack of familial influences underlying attributional style implies that attributional style is not a mediator of genetic influences on depression, which supports the finding that attributions are largely an outcome of current mood rather than a causal influence on depression. This reasoning is also backed up by comparisons of the non-depressed siblings of participants with depression with the non-depressed siblings of control group members, because no difference in attributions was seen despite the former group being at higher risk of developing depression in the future. A previous population study of adolescents (mainly twin pairs plus some sibling pairs) did find evidence of some genetic as well as substantial non-shared environmental influences on attributional style, ${ }^{5}$ implying that there are genetic influences on variation in attributional style at younger ages. However, the point estimates of the adolescent pairs' correlations were lower for the sibling pairs than for the twin pairs, and were lower for opposite-gender than for same-gender pairs, suggesting the importance of the contextual factors of age and gender. This may explain the lack of familial influences found in the current study: everyday environments of grown-up siblings tend to be quite dissimilar, whereas the environments of siblings in childhood tend to be very similar (especially for twin pairs, who are the same age and are more often of the same gender than are sibling pairs). This is consistent with a large proportion of individual differences in attributional style being due to aspects of an individual's current environment and current mood, rather than being enduring traits that can reliably predict future depression.

\section{Limitations of the study}

Limitations of the study include the lack of systematic ascertainment of probands and controls. The control proband group was selected for health, so some findings may relate to these participants being 'super-healthy'. The study included relatively few participants who had experienced depression in the past but who were currently healthy, reducing power to detect long-lasting influences of previous depressive illness on attributional style.

\section{Implications}

The way in which individuals attribute their experiences may be less of a risk factor and more of a symptom of depression than previously thought. Past episodes of depression may produce long-lasting negative attributions relating to the self, in addition to other pessimistic attributions that are linked to both observed and self reported current depression. It is therefore important to look out for and address these pessimistic attributions in people with recurrent depression.

Harriet A. Ball, MSC, Peter McGuffin, PhD, FRCP, FRCPsych, Anne E. Farmer, MD FRCPsych, Medical Research Council Social Genetic and Developmental Psychiatry Centre, Institute of Psychiatry, London, UK

Correspondence: Harriet Ball, Box 080, MRC Social Genetic and Developmenta Psychiatry Centre, Institute of Psychiatry, De Crespigny Park, London SE5 8AF, UK. Email: harriet.ball@iop.kcl.ac.uk

First received 13 Apr 2007, final revision 6 Dec 2007, accepted 8 Jan 2008

\section{References}

1 Beck AT. Depression: Clinical, Experimental, and Theoretical Aspects. Harper \& Row, 1967

2 Abramson LY, Metalsky GI, Alloy LB. Hopelessness depression: a theorybased subtype of depression. Psychol Rev 1989; 96: 358-72.

3 Sweeney PD, Anderson K, Bailey S. Attributional style in depression: a metaanalytic review. J Pers Soc Psychol 1986; 50: 974-91.

4 Alloy LB, Abramson LY, Whitehouse WG, Hogan ME, Panzarella C, Rose DT. Prospective incidence of first onsets and recurrences of depression in individuals at high and low cognitive risk for depression. J Abnorm Psychol 2006; 115: 145-56.

5 Lau JFY, Rijsdijk FV, Eley TC. I think therefore I am: a twin study of attributional style in adolescents. J Child Psychol Psychiatry 2006; 47 696-703.

6 Farmer A, Harris T, Redman K, Sadler S, Mahmood A, McGuffin P. The Cardiff Depression Study: a sib-pair study of life events and familiality in major depression. Br J Psychiatry 2000; 176: 150-5.

7 World Health Organization. The ICD-10 Classification of Mental and Behavioural Disorders. Diagnostic Criteria for Research. WHO, 1993.

8 Wing JK, Babor T, Brugha T, Burke J, Cooper JE, Giel R, Jablenski A, Regier D, Sartorius N. SCAN: Schedules for Clinical Assessment in Neuropsychiatry. Arch Gen Psychiatry 1990; 47: 589-93.

9 Brown WG, Harris TO. Social Origins of Depression. A Study of Psychiatric Disorder in Women (5th edn). Brown \& Harris, 1978.

10 Tennant $C$, Smith A, Bebbington P, Hurry J. The contextual threat of life events: the concept and its reliability. Psychol Med 1979; 9: 525-8.

11 Beck AT, Ward $\mathrm{CH}$, Mendelson M, Mock J, Erbaugh J. An inventory for measuring depression. Arch Gen Psychiatry 1961; 4: 561-71.

12 Beck AT, Steer RA. Internal consistencies of the original and revised Beck Depression Inventory. J Clin Psychol 1984; 40: 1365-7.

13 Peterson C, Semmel A, von Baeyer C, Abramson LY, Metalsky Gl, Seligman MEP. The Attributional Style Questionnaire. Cognit Ther Res 1982; 6: 287-99.

14 Peterson C, Seligman ME. Causal explanations as a risk factor for depression: theory and evidence. Psychol Rev 1984; 91: 347-74.

15 Khan AA, Jacobson KC, Gardner CO, Prescott CA, Kendler KS. Personality and comorbidity of common psychiatric disorders. Br J Psychiatry 2005; 186: 190-6.

16 Kendler KS, Neale MC, Kessler RC, Heath AC, Eaves L. A longitudinal twin study of personality and major depression in women. Arch Gen Psychiatry 1993; 50: 853-62.

17 Farmer AE, McGuffin P. Humiliation, loss and other types of life events and difficulties: a comparison of depressed subjects, healthy controls and their siblings. Psychol Med 2003; 33: 1169-75.

18 Farmer A, Redman K, Harris T, Mahmood A, Sadler S, McGuffin P. Sensationseeking, life events and depression: the Cardiff Depression Study. $\mathrm{Br} J$ Psychiatry 2001; 178: 549-52. 